\title{
A computational framework for the design and robotic manufacturing of complex wood structures
}

\author{
Roberto Naboni ${ }^{1}$, Anja Kunic ${ }^{2}$ \\ ${ }^{1}$ CREATE Group, University of Southern Denmark (SDU) - Unit of Civil and Ar- \\ chitectural Engineering ${ }^{2}$ Politecnico di Milano \\ ${ }^{1}$ ron@iti.sdu.dk²anja.kunic@gmail.com
}

\begin{abstract}
The emerging paradigm of Industry 4.0 is rapidly expanding in the AEC sector, where emergent technologies are offering new possibilities. The use of collaborative robots is enabling processes of advanced fabrication, where humans and robots coexist and collaborate towards the co-creation of new building processes. This paper focuses on setting a conceptual framework and a computational workflow for the design and assembly of a novel type of engineered wood structures. The aim is advancing timber construction through complex tectonic configurations, which are informed by logics of robotic assembly, topology and material optimization, and combinatorial design. Starting from the conceptualization of robotic layered manufacturing for timber structures, this work presents the development of a digital twin applied to the voxel-based design of complex timber structures.
\end{abstract}

Keywords: Digital Materials, Robotic Assembly, Wood structures, Voxel-based design, Topology Optimization

\section{INTRODUCTION}

Current technological advancements, combined with increasingly complex environmental issues, are challenging architects, designers, civil and robotic engineers to seek for novel solutions in making the discipline of architecture and the construction industry more efficient, automated and informed (Willmann et al. 2018). In particular, the integration of robots with computational design techniques enables a radical change and enrichment of the physical nature of architecture, where material properties and organization is the main design driver, leading to the emergence of a phenomenon described by Gramazio and Kohler as digital materiality (2008). Through robotics and (computationally) controlled material information, the architectural making has opened up entirely new aesthetics and functional potentials. Recently, researchers have shown an increased interest in exploring digital materials in construction (Retsin 2019; 2018; 2016), driven by the ability to process, manipulate and embed a large amount of information, so-called big-data into the design, fabrication and assembly processes (Carpo 2017). As argued by Gershenfeld et al. (2015), in order to be considered digital, a fabrication process is based on the assembly of fundamentally digital materials, which allow the exact specification of material configuration, and for the detection and correction of eventual errors in 
that configuration (Popescu, 2007).

Inscribed in the paradigm of Industry 4.0, this research focuses on the implementation of a computational framework that integrates simulation, design, fabrication and assembly of complex engineered wood structures, extending to the realm of constructions the concept of Digital Twin, intended as a continuous virtual support to all phases of the product life cycle (Rodič 2017). In this process, we start from a robotic fabrication setup to inform the development of efficient timber structures, integrated with notions of combinatorial design, digital materiality, and performance.

\section{METHODOLOGY}

An assembly-aware process for integrated wooden structures is proposed here as a computational evolution of composite engineered wood. This is used in constructions to achieve specific or higher strength to mass timber members (APA 2008). As a material, this is manufactured from smaller size wood layers that are glued together and positioned with fibres oriented in a functional arrangement. Glue Laminated wood (Glulam) is mostly used for beam-like elements, where wood lamellas have grains aligned parallel to the length of the piece. Cross-laminated timber is instead made of cross-wise elements, which compensate anisotropy, and thus have preferable applications in walls, floors and roofs elements (Stora Enso 2017). With this work, we challenge engineered wood by looking at synergistic opportunities in construction automation by the use of flexible robot cells, topology optimization and voxel-based design to enable the construction of highly articulated and structurally optimized wooden structures. The aim is to develop a workflow for the design and production of efficient and highly specified tectonic configurations. develop a workflow for the design and production of efficient and highly specified tectonic configurations.

We envision a robotic setup consisting of flexible and reconfigurable cells, where collaborative robots are employed in a novel process of Layered Manu- facturing, to select, pick, place and connect individual wood elements. This concept implements logics of Additive Manufacturing to large scale timber construction: printed layers are here replaced by woodbased digital materials which are positioned incrementally in space, and combined together to form larger objects. This approach allows building complex and high-resolution timber elements with small scale robots. In comparison with additive processes, material properties can be tuned extensively by the definition of relevant attributes which can provide control over intensive and extensive properties of an overall structure, with possible variations in space (Fig. 1).

The computational workflow for the design and manufacturing of voxel-based engineered structures is developed within the Grasshopper environment, where design, analysis, and manufacturing routines are converging to guarantee a continuous and integrated flow of information.

\section{Voxel Attributes}

Digital Materials are defined by a discrete set of components, with a defined number of allowable relative positions and orientations, and the explicit control of the placement of individual components. (Popescu, 2007). In order to translate digital information into physical building blocks, it is fundamental to define voxel attributes within the computational environment, which will then subserve the combinatorial design and the physical realization of digital materials. With the aim to produce highly complex and structurally optimized structures, several material attributes are defined. In accordance with the assembly process outlined previously, we outline a combinatorial matrix with four fundamental attributes to describe specific tectonic characteristics of digital building blocks: material class, orientation, connectivity, and assembly direction (Fig.2).

Material Strength - In glulam elements, layers of material with different strength class are often combined, where the high performing is placed in zones with maximum tensile and compressive stress, 
Figure 1

Layered robotic assembly of digital building blocks

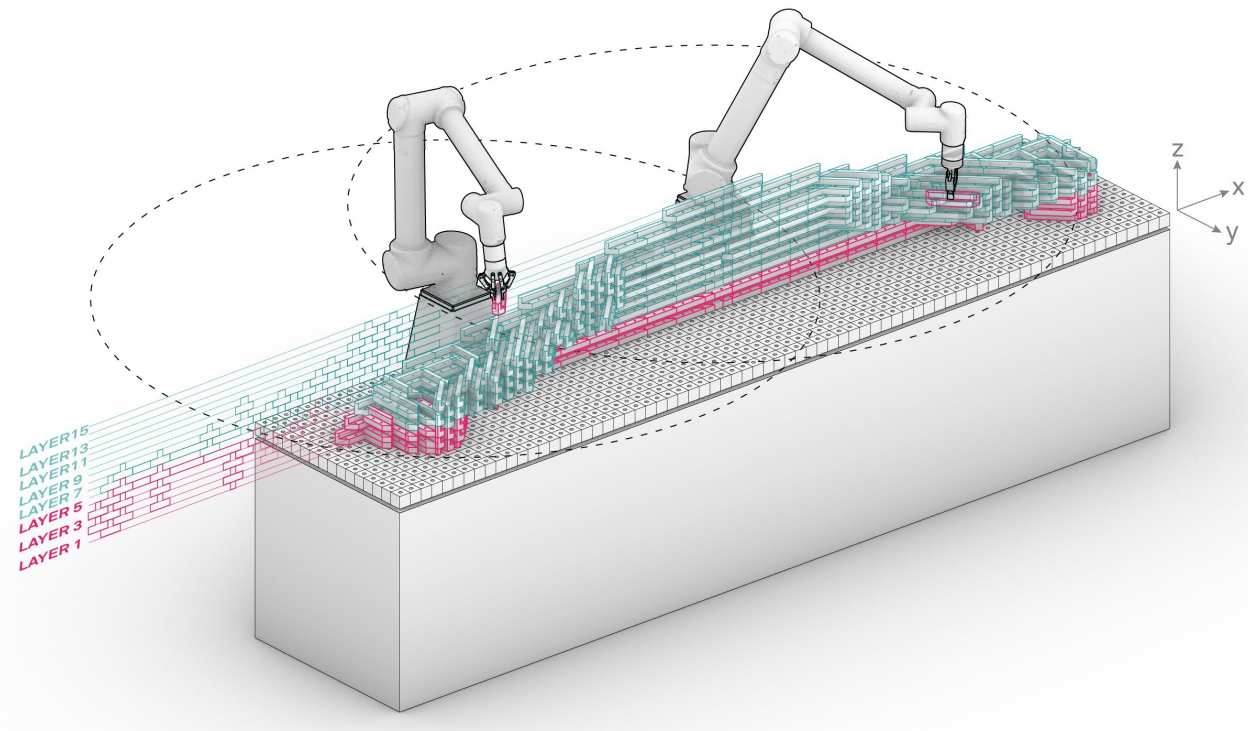

in order to make more efficient use of the material. In this case, we limit our description to a scalar strength value which will be later compared to existing stresses in the structure, in order to determine the appropriate class of material and improve weight and cost efficiency in the structure.

Orientation - Wood is an anisotropic material, therefore it has properties varying in different directions. Controlling the orientation of wood is essential to achieve efficient structural behaviour. In comparison with typical engineered wood, we aim at providing higher freedom in orienting wood grains to conceive topologically convoluted structures, with coherent performance in material, form and structure. In this specific project, we align wood fibres parallel to the load path trajectories in the structure, discretized into a set of vectors snapped to angles of $0^{\circ}$ $45^{\circ}, 90^{\circ}$ in order to reduce the conveniently the number of existing options.
Voxel Connectivity - Mechanical behaviour of a discrete structure is highly dependent on the interconnectivity between its parts, which is preferably defined in coherence with the forces flowing from the applied load to the constraints of a structure. Connectivity can be then described in the three coordinates $X, Y, Z$, involving a large number of potential combinations. In this specific project, we assume a constant connectivity vector in the Z-direction, given the layered addition of pieces, and possible vectors which are combined in the XY plane with a total of 56 possible spatial orientations. This can be synthesized in four component oriented in various angles.

Assembly - A voxel description reflects conditions emerging from fabrication and especially robotic assembly processes. In this specific case, the layered manufacturing processes determine $Z$ as the assembly direction accessed by the robot, which ensures a path planning free of collisions in order to grip and 
place the blocks. The two remaining axes, $X$ and $Y$ will then be specified as complementary connection axis, mostly relying on morphological descriptors to ensure further assembly properties.

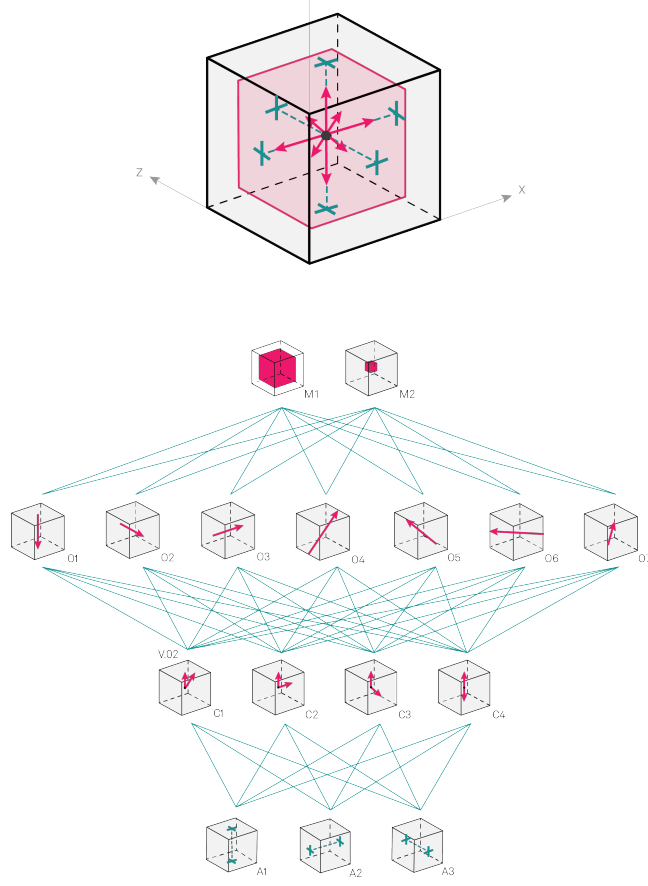

\section{From Voxel to Digital Material}

The description of voxel attributes is actualized into a construction kit, i.e. a set of digital materials which are providing a physical representation of an abstract and generalized definition. These digital materials are allocated in space to form meaningful tectonic configurations which follow various instances, informed by a global level of design, analysis and optimization explained in the next paragraph.

In our approach, clusters of connected voxels are forming digital materials made of different wood types, according to the needed strength class required within a structure, and its availability. The shape of the individual blocks reflects the layered process of robotic assembly. In the XY plane, the main axis of each block is characterized by a 'gripping zone', which allows the robot to approach freely and access individual components along the process. In the same plane are placed holes to provide connectivity along the Z-axis.

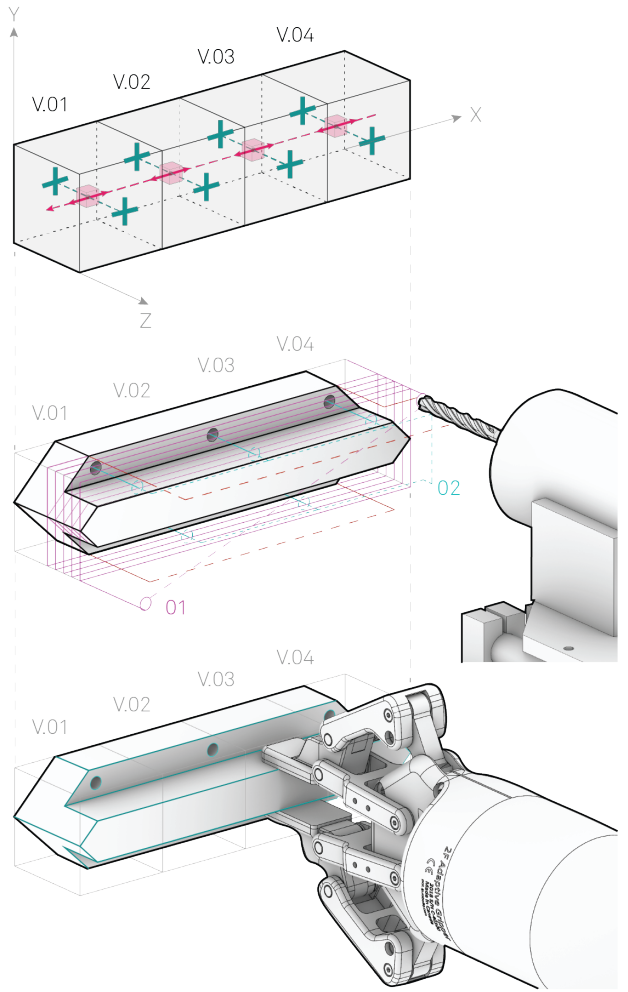

The shape ends in an arrow-like profile which is used as a converging area to provide self-alignment in the assembly process, as well as ensuring shear resistance in the final structure. Blocks are positioned into stacked layers where their $T$ section provides a mech-
Figure 2

Design attributes matrix for characterization of digital materials

Figure 3

Characterization of digital building block through defined design attributes: digital description of the voxels; CNC milling toolpath for the block fabrication; robotic gripping principle of the block 
Figure 4

Computational workflow from structural analysis to discretized structure anism for a lateral load transfer.

Building blocks are manufactured through CNC milling of off-shelf wood studs with 50 by $50 \mathrm{~mm}$ cross-section. A discrete set of elements is produced from the same original piece and transformed into a final shape optimized to minimize material waste and machining time. The block can be gripped at any point of its length, manoeuvred, and connected to the others without self dependencies (Fig. 3).

\section{Voxel-based Design and Multi-Material Op- timization}

After the introduction of local attributes in voxel and digital materials, we focus on a global level of design and optimization of a hybrid structural wood system. This is developed and then discretized into a voxelbased description to provide meta-information on the fields governing the spatial allocation of digital materials.

The computational process begins with a Finite Element Analysis (FEA) and Topology Optimization (TO) run using Millipede as the main calculation engine, and Monolith for the voxel-based stresses interpretation. In this experiment, we focus on a hybrid bridge-like structure, with enlarged midsection, spanning $600 \mathrm{~cm}$. Initial boundary conditions are set applying a distributed live load of $2.5 \mathrm{kN} / \mathrm{m}^{2}$ and supports. Emerging information about the load path is obtained from the FEA to visualize the way in which loads flow in the structure, from the point of application to the point where they are reacted (Kelly and Tosh 2000). In parallel, a multi-material TO based on Von Mises criteria is run targeting a volume reduction of $60 \%$, starting from an initial volume which represents the bounding design space. Resulting from this optimization is a discretized structural layout, which is computed with a voxel definition corresponding with the voxel size utilized in the digital materials and assembly setup. With this operation, areas of highest stress concentration define an intricate and hierarchical structure with complex topology (Fig.4).

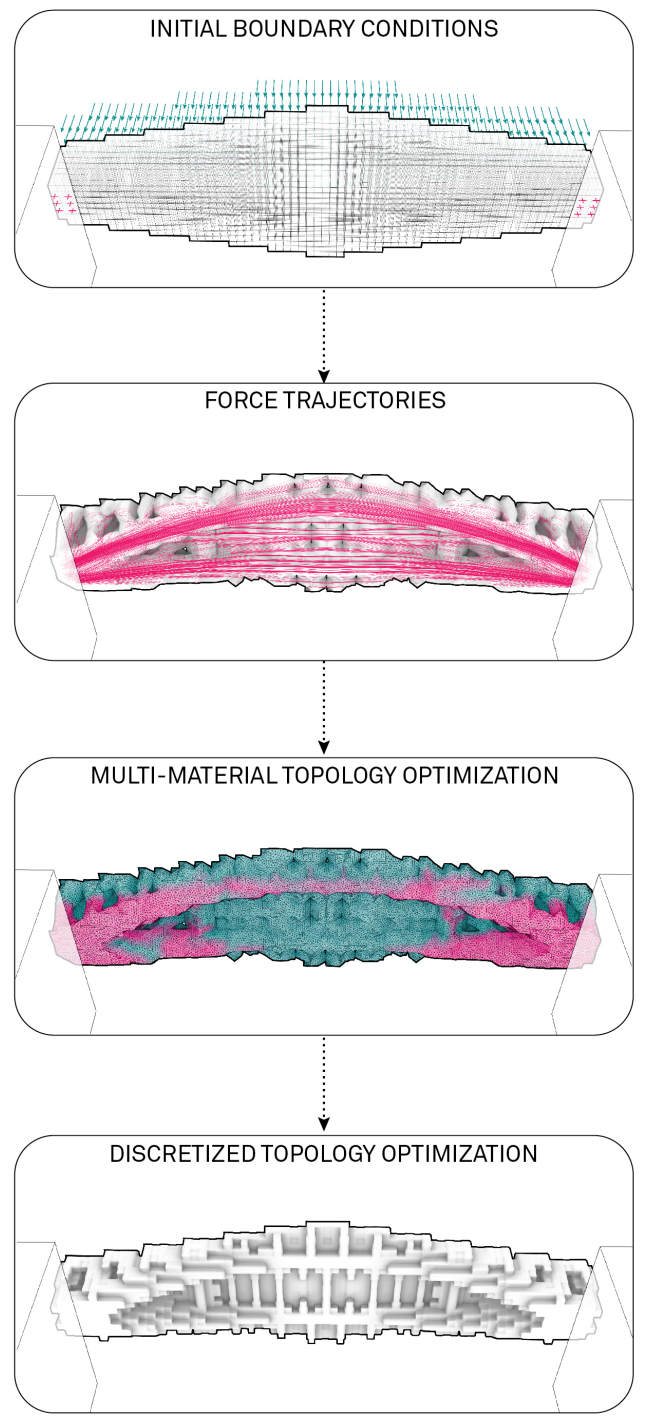




\section{Performance-based Design Discretization}

The outcome from the structural design and optimization are interpreted and described in reference to the previously outlined voxel attributes. Digital data embedded within the voxels are sorted and grouped based on the similarity of the mixed attributes and the closeness to similar types. Further on, these clusters are translated into a set of building blocks, whose topology, geometry and aggregation logic reflects the attributes by which they have been defined.

The primary attribute which influences the orientation of the blocks aggregation and therefore the directionality of the wood fibres is the field description arising from the load path trajectories, evaluated in each voxel. Angles are discretized into four groups with values of $0^{\circ}, 45^{\circ}, 90^{\circ}, 135^{\circ}$ (Fig.5a). Secondly, the voxel connectivity in the structure is computed, in coherence with the stress flows, to ensure that the actual discretized components provide continuous load transmission up to the structural supports. As previously mentioned, combinations of voxel connectivity are varying along the $X Y$ plane, while the connectivity along the Z-axis is assumed as a constant, predefined with the layered manufacturing process. The discretized description is based on four types of components, with variable orientation in space (Fig.5b).

Finally, a description of the specific material class of the wood pieces is applied. Based on the stress distribution along the structure, a specific strength of wood is defined. In this example C24 in highly stressed areas, and $\mathrm{C} 16$ in areas with lower stress values, similarly to the glulam construction (Fig.5c).

\section{RESULTS}

The paper describes a computational framework for the automated design, fabrication, and robotic assembly of complex engineered wood structures. The workflow is developed to provide a digital twin to support the whole life-cycle of such structures, involving the integration of combinatorial design strategies, performance simulation, and voxel-based

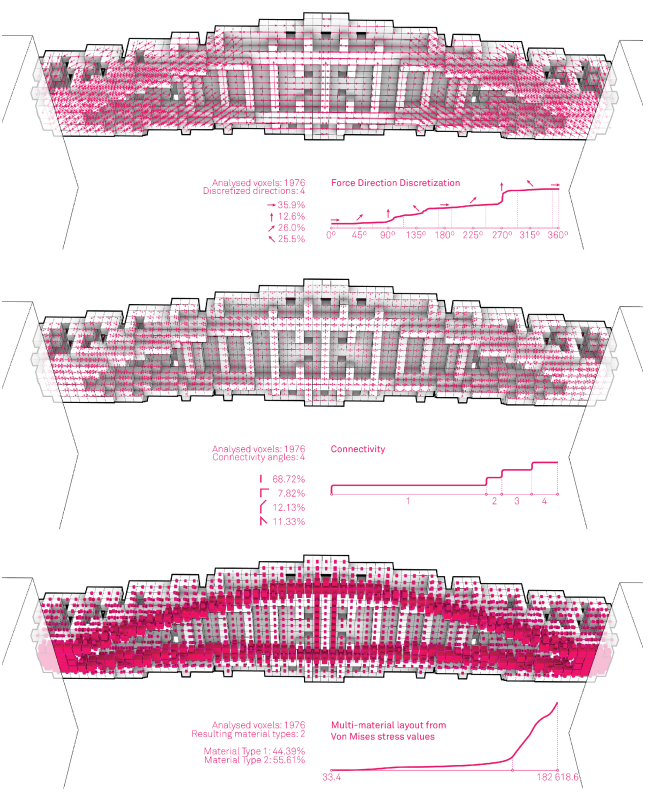

Figure 5

Discretized load paths, voxel connectivity and multi-material layout informed by the Topology Optimization and FEA outcomes design to achieve a highly controlled material organisation. From the application of digital materials to the design of an experimental structure emerge different results. The use of discrete construction elements permits to describe complex hierarchical structures from a very limited set of construction components, reconducted to six main typologies, with precise allocation in space (Fig.6). This process integrates optimization logics which contribute to achieving different results, such as the reduction in the volume and material employed by $60 \%$. From the discretization of the load path into predefined angles, an average deviation of only $13.28^{\circ}$ is obtained, while reducing drastically the overall components inventory. Moreover, the internal connectivity among components ensures uninterrupted load paths. Finally, the structural efficiency is increased with the use of multi-material TO: higher and lower strength material is used to build respectively $44.39 \%$ and $55.61 \%$ of the overall structure. This combination guarantees a $10 \%$ reduction in weight and related reduction in material costs. 
Figure 6

Spatial distribution layout of six different types of building blocks
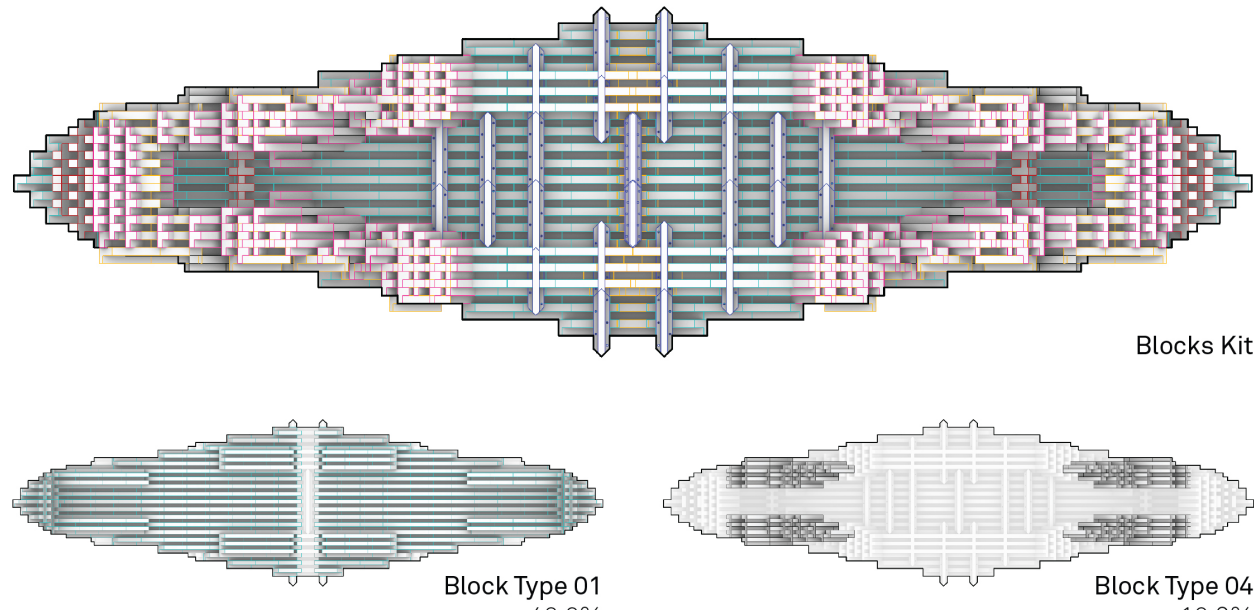

$42.6 \%$
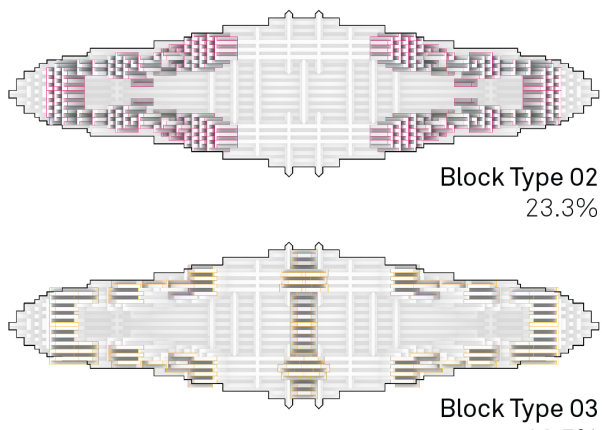

$12.5 \%$

Beyond quantitative results, the structural design emerges as a physical interpretation of big data, as the use of digital materials reveals emerging tectonic attributes at the global scale, which are an expression of the computational methods used to calculate them. This methodology allows achieving hierarchical and highly articulated structural configurations through a sustainable process of discrete automation (Fig.7).

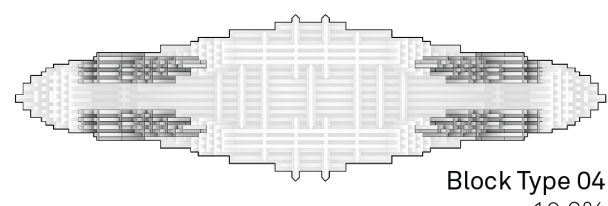

$10.9 \%$

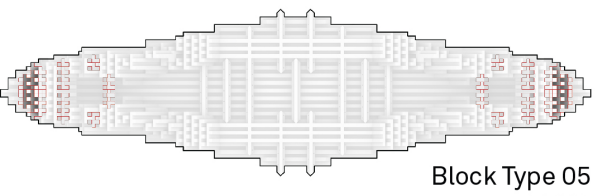

$7.9 \%$

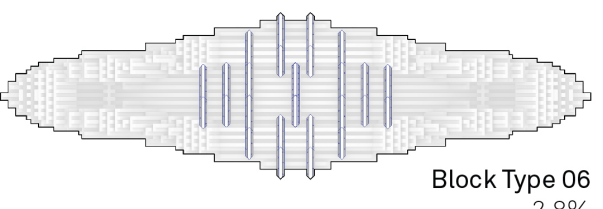

$2.8 \%$

\section{CONCLUSIONS}

Following this initial investigation into the computational framework for complex engineered timber structures, several further developments are envisioned in the following areas: the robotic assembly of the structure through the use of collaborative robots and flexible cells; the mechanical testing of different connection types among the components; the realization and testing of a full-scale demonstrator to evaluate the efficiency of the manufacturing system, and validate the structural performance. 


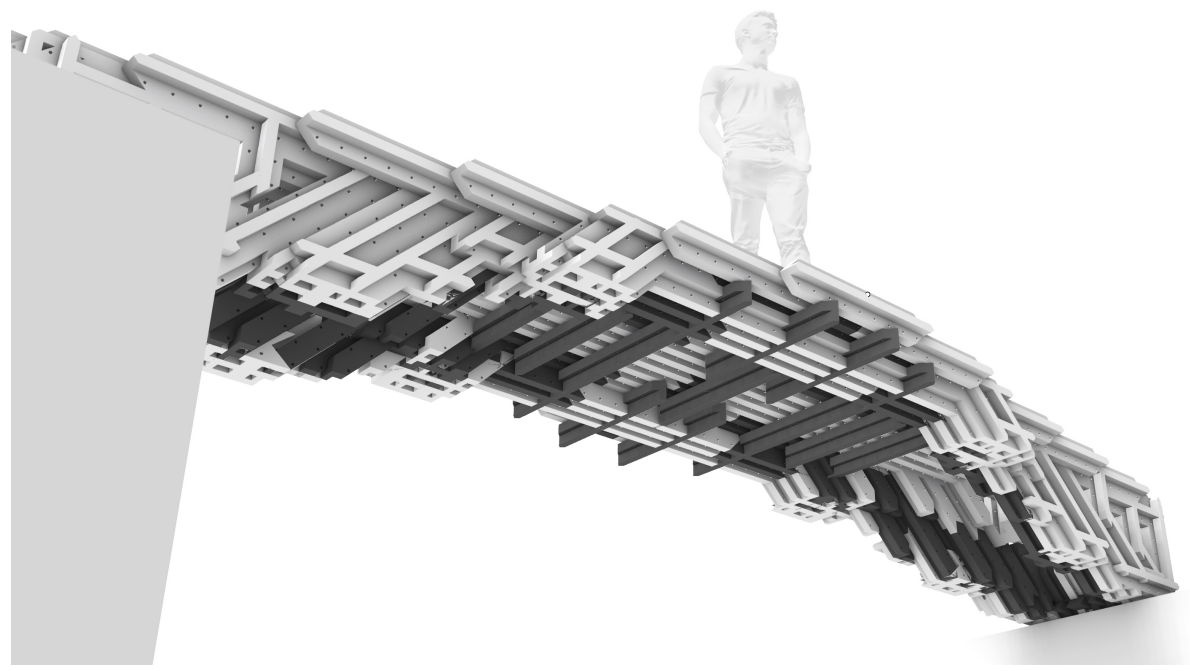

Figure 7

Multi-material,

topologically optimized bridge structure

\section{ACKNOWLEDGEMENTS}

The authors wish to thank Takwa El Gammal for her contribution to the research development and the support in preparing the graphics used in the article.

\section{REFERENCES}

APA - The Engineered Wood Association, initials missing 2008, Glulam product guide, APA Headquarters, 2008

Carpo, M 2017, The second digital turn : design beyond intelligence, MIT Press

Gerschenfeld, N, Carney, M, Bennett, B, Calisch, S and Wilson, $S$ 2015, 'Macrofabrication with Digital Materials: Robotic Assembly', Architectural Design, 85(5), pp. 122-127

Gramazio, F and Kohler, M 2008, Digital Materiality in Architecture, Lars Muller

Kelly, DW and Tosh, MW 2000, 'Interpreting load paths and stress trajectories in elasticity', Engineering Computations, $17(2)$, pp. 117-135

Popescu, G 2007, Digital materials for digital fabrication, Master's Thesis, Massachusetts Institute of Technology

Retsin, G 2016 'Discrete Assembly and Digital Materials in Architecture', Proceedings of eCAADe 2016

Retsin, G 2018, 'Digital Material. Material and Novelty', MAJA. KEVAD 2018
Retsin, G (eds) 2019, Reappraising the digital in architecture, AD. Architectural Design

Rodič, B 2017, 'Industry 4.0 and the New Simulation Modelling Paradigm', Organizacija, 50(3), pp. 193207

Varga, C, Guntschnig, G and Horx-Strathern, O (eds) 2017, The future of Timber Construction CLT - Cross Laminated Timber. A study about changes, trends and technologies of tomorrow, Zukunftsinstitut $\mathrm{GmbH}$

Willmann, J, Block, P, Hutter, M, Byrne, K and Schork, T (eds) 2018, Robotic Fabrication in Architecture, Art and Design 2018, Springer Nature Switzerland 